\title{
Confidence in the prediction of neurodevelopmental outcome by cranial ultrasound and MRI in preterm infants
}

\author{
Phumza Nongena, ${ }^{1,2}$ Ash Ederies, ${ }^{1,2}$ Denis V Azzopardi, ${ }^{1,2}$ \\ A David Edwards ${ }^{1,2}$
}

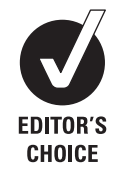

\section{INTRODUCTION}

Preterm birth is increasing and the rate of neurodevelopmental impairment in survivors remains high. ${ }^{2}$ Clinicians routinely need to provide parents and carers with prognostic information for their vulnerable infants, and most do this with the aid of some form of neuroimaging. Cranial ultrasound is cheap, safe and can be performed at the cot side by the attending neonatologist or paediatric radiologist. MRI is less widely available, more expensive and requires transportation to an imaging unit, but provides anatomically richer data. However, it is not clear how comparable the images are, nor can we be sure about the predictions made by either imaging method.

In September's issue of the journal, the analysis by Horsch et a ${ }^{\beta}$ of contemporaneously acquired cranial ultrasound and $\mathrm{MR}$ images at term corrected age in a cohort of infants born at $<27$ weeks gestation found close concordance between the two imaging methods, with only marginal, if any, additional information with MRI. However imaging findings were normal or showed mild MRI changes in $>80 \%$ of infants and only $3 / 72$ (4\%) showed severe abnormality, reducing confidence in any comparison. In fact the prognostic power of each imaging modality was undefined as neurodevelopmental outcome was not reported.

To provide practicing clinicians with pragmatic estimates of the confidence

\footnotetext{
${ }^{1}$ Institute of Clinical Sciences, Imperial College London and MRC Clinical Sciences Centre, Hammersmith Hospital, London, UK

${ }^{2}$ Division of Neonatology, Imperial College Healthcare NHS Trust, London, UK

Correspondence to A David Edwards, Department of Paediatrics, Hammersmith Hospital, Du Cane Road, London W12 ONN, UK;

David.Edwards@imperial.ac.uk
}

limits appropriate when interpreting cranial ultrasound and MRI, we searched the literature for studies that allowed reasonable quantitative estimates of prognostic value, ensuring that repeated publications of individuals were excluded by contacting researchers directly where necessary. From the surprisingly small number of suitable studies we selected and aligned information as objectively as possible with familiar imaging and neurodevelopmental outcome classifications, with outcomes, usually determined at about 2 years of age, categorised broadly as abnormal neuromotor development (estimated as the presence of cerebral palsy or a low Bayley Psychomotor Developmental Index of below 70) or cognitive impairment (estimated as a low Bayley Mental Developmental Index of below 70 or a Griffiths Developmental Quotient of below 85).

To combine multiple studies into single estimates, we used Meta-Disc4 software $^{4}$ to calculate pooled likelihood ratios with $95 \%$ CIs and then applied a Bayesian approach to calculate the pooled probability, together with $95 \%$ CIs, that particular imaging appearances would be associated with specific neurodevelopmental outcomes. Bayesian analysis allows a background probability (called the prior probability) to be modified by new information from a test to provide an updated post-test or posterior probability. We used the overall background risks described in the recent Epipage study ${ }^{5}$ as the prior probability and calculated the post-test probability given the neuroimaging result.

We offer these results as positive predictive values (PPV; the proportion of subjects with a positive test result who have the outcome being tested for) or the pooled post-test probability (the probability that a patient with a given test result will have a particular neurological outcome) together with 95\% CIs for the estimates. These data should indicate to clinicians how much confidence can be placed in a prognosis assigned after neuroimaging.

\section{CRANIAL ULTRASOUND}

Images were interpreted as normal if there was no haemorrhage in the germinal matrix, ventricles or brain tissue, no evidence of brain tissue destruction and no marked ventricular dilatation. Periand intraventricular haemorrhage (IVH) was classified according to the scale of 1-4 after Papile et a $1^{6}$ or the broadly similar scale developed by Volpe ${ }^{7}$ with cerebellar haemorrhage considered separately. We grouped together images that suggested focal or multifocal tissue destruction due to cystic periventricular leukomalacia (PVL). In the absence of precise measurements in many studies, we made pragmatic decisions on defining moderate and severe ventricular dilation. The probabilities of cerebral palsy associated with specific imaging findings are given in table 1, together with the $95 \%$ CIs for those estimates.

\section{Normal scan}

A series of studies over a 30-year period have shown that a normal ultrasound scan provides considerable confidence that an infant will have normal neuromotor development. The predictive accuracy is high and confidence limits narrow: in one typical study the PPV was $99 \%(95 \% \text { CI } 98 \% \text { to } 99 \%)^{8}$; combining suitable studies, the pooled probability for normal outcome was $94 \%$ (95\% CI $92 \%$ to $96 \%),{ }^{8-12}$ although heterogeneity between studies was high $\left(\mathrm{I}^{2}\right.$ $88 \%$ ). Cognitive impairment is excluded slightly less effectively: in a typical large study, a normal ultrasound scan predicted normal cognitive function with a PPV of $77 \%$ (95\% CI $74 \%$ to $80 \%),{ }^{13}$ and the pooled probability of a normal cognitive outcome with a normal ultrasound scan is $82 \%$ (95\% CI $79 \%$ to $85 \%) .{ }^{11} 13$

\section{Grade 1 or 2 IVH}

In one major study, images with only grade 1 or 2 IVH showed a low risk of abnormal neuromotor development with narrow confidence limits: PPV 6\% (95\% CI $4 \%$ to $9 \%$ ). ${ }^{9}$ Combining studies together produced broadly similar findings but with wider confidence limits, the pooled probability of abnormal neuromotor development being 9\% (95\% CI $4 \%$ to $22 \%) .{ }^{914}$ 
Table 1 Prediction of abnormal neuromotor function by cranial ultrasound

\begin{tabular}{|c|c|c|c|c|}
\hline \multirow[b]{2}{*}{ Ultrasound test result } & \multicolumn{4}{|l|}{ Cerebral palsy } \\
\hline & Pre-test probability & Likelihood ratios $(95 \% \mathrm{CI})$ & Post test probability (95\% CI) & Heterogeneity among studies (I2) \\
\hline Normal scan & $9 \%$ & $0.5(0.4$ to 0.7$)$ & $5 \%(4 \%$ to $6 \%)$ & $90 \%$ \\
\hline Grade 3 IVH & $9 \%$ & $4(2$ to 8$)$ & $26 \%(13 \%$ to $45 \%)$ & $82 \%$ \\
\hline Grade 4 haemorrhage (any) & $9 \%$ & 11 (4 to 31 ) & $53 \%(29 \%$ to $76 \%)$ & $84 \%$ \\
\hline Cystic PVL & $9 \%$ & $29(7$ to 116$)$ & $74 \%(42 \%$ to $92 \%)$ & $90 \%$ \\
\hline
\end{tabular}

\begin{abstract}
Normal scan refers to absence of haemorrhage within the brain parenchyma or ventricles, cysts or ventricular dilation. The grade of IVH (intraventricular haemorrhage) is given according to the Papile classification. PVL indicates periventricular leukomalacia. Ventricular dilation indicates moderate to severe ventricular dilation not meeting the criterion for hydrocephalus. Hydrocephalus indicates massive ventricular dilation $>4 \mathrm{~mm}$ above the 97 th centile. Pre-test probability refers to the prevalence of cerebral palsy based on the Epipage study. ${ }^{4}$ The likelihood ratio is the probability that a patient with cerebral palsy has a positive test (abnormal ultrasound result). Post-test probability is the probability that a patient with a specific abnormality on cranial ultrasound will have abnormal neuromotor function. Heterogeneity is a measure of similarity between studies and the validity of statistical pooling.
\end{abstract}

\section{Grade 3 IVH}

In one typical study, the presence of grade 3 IVH was associated with a modest increase in the risk of abnormal motor development but wide confidence limits, with PPV $24 \%$ (95\% CI $12 \%$ to $42 \%$ ). ${ }^{8}$ However, combining studies produced a pooled probability of abnormal motor development being $26 \%$ (95\% CI $13 \%$ to $45 \%) .811$

\section{Grade 4 IVH}

In a typical large study, these lesions (also called parenchymal haemorrhagic infarction or cerebral venous infarction) predicted abnormal motor development with an appreciably increased risk PPV of $47 \%$ (95\% CI $31 \%$ to $64 \%) .{ }^{8}$ The pooled probability for abnormal motor development estimated by combining suitable studies was $53 \%$, but again with wide confidence limits $(95 \%$ CI $29 \%$ to $76 \%)$, probably in part due to the variability in both site and size of lesions. ${ }^{81011}$

\section{Cystic PVL}

In a typical large study, images showing cystic PVL were predictive of cerebral palsy with a PPV of $77 \%$ (95\% CI 59\% to $89 \%)$, although the rarity of the finding was reflected by wide confidence limits. ${ }^{8}$ The combination of studies suggested that the pooled probability for abnormal neuromotor outcome with cystic PVL is $74 \%$, but again there is considerable uncertainty in individual cases $(95 \%$ CI $42 \%$ to $92 \%)^{89}$

\section{Ventricular dilatation}

Definitions of moderate and severe ventricular dilatation differ widely in the literature. We defined ventricular dilatation pragmatically, and ventricular dilatation at or near term was modestly predictive of major disability. In one typical study, the PPV was $27 \%$ (95\% CI $15 \%$ to $43 \%),{ }^{15}$ while the pooled probability for abnormal motor development was $22 \%$ (95\% CI $17 \%$ to $28 \%)^{13} 15$

\section{Post haemorrhagic hydrocephalus}

The outcome for children with more precisely defined post haemorrhagic hydrocephalus is also variable. In the selected group of patients in the recent Drift study, ${ }^{16}$ the children receiving standard treatment could be predicted to have abnormal neuromotor function with PPV of $69 \%$ (95\% CI $50 \%$ to $83 \%$ ). Combining studies together produced a lower estimate but emphasised this uncertainty: the pooled probability of abnormal neuromotor outcome was $27 \%$ (95\% CI $10 \%$ to $56 \%) .{ }^{111} 16-18$

\section{Cerebellar haemorrhage}

Cerebellar haemorrhages are not commonly reported. They are often detected in association with supratentorial lesions although they can rarely occur in isolation. In the previous issue of the journal, Horsch et al found that ultrasound failed to detect cerebellar lesions. ${ }^{3}$ However, O'Shea et al found that the presence of cerebellar haemorrhage on ultrasound predicted abnormal neuromotor outcome, but with wide CIs: PPV 71\% (95\% CI $42 \%$ to $90 \%){ }^{13}$

\section{MAGNETIC RESONANCE IMAGING}

Relatively few studies have attempted to determine the value of MRI for predicting abnormal motor development or cognitive impairment ${ }^{19-23}$ and, like the ultrasound studies, they use different imaging and outcome criteria. We have again pragmatically selected and aligned results, focusing on studies using widely available MR techniques.

\section{White matter abnormalities}

Woodward et al evaluated the presence of white matter injury using a combination of imaging appearances to predict longterm neurodevelopmental outcome. ${ }^{21}$ Using a white matter grading score ranging from normal to moderate-severe, the PPV of moderate-severe white matter abnormalities for abnormal motor development was $31 \%$ (95\% CI $17 \%$ to $49 \%)$ and for cognitive impairment was $34 \%$ (95\% CI $20 \%$ to $52 \%$ ). Combining studies with reasonable overlap between the image categorisations suggested that moderate-severe white matter abnormalities predicted abnormal neuromotor development with a pooled probability of $35 \%$ (95\% CI $19 \%$ to $55 \%),{ }^{20} 21$ and cognitive impairments with a pooled probability of $52 \% \quad(95 \%$ CI $36 \%$ to $67 \%)^{2123}$

\section{Ventricular enlargement}

In one study, ventricular enlargement with a ventricular diameter $>8 \mathrm{~mm}$ predicted long-term neurodevelopmental impairment with a PPV of $86 \%(95 \%$ CI $42 \%$ to $99 \%) .{ }^{23}$ Another study found that a combination of ventriculomegaly and white matter abnormality predicted abnormal motor development with a PPV of $55 \%(95 \%$ CI $23 \%$ to $85 \%) .{ }^{22}$ Unfortunately, the diagnostic categories used prevented combination of these data, and the wide confidence limits suggest caution in the clinical application of these data.

\section{Abnormalities of the posterior limb of the internal capsule}

When a supratentorial lesion is visualised, evaluation of the posterior limb 
of the internal capsule (PLIC) might be expected to improve diagnosis of motor deficits because of its importance as a conduit of motor signalling. One group has suggested that in the presence of PVL, an abnormal appearance of the PLIC predicts abnormal motor development with a PPV of $90 \%$ (95\% CI 54\% to 99\%), and with IVH a PPV of $78 \%(95 \%$ CI $40 \%$ to $96 \%$ ), although the small number of patients studied means that the confidence limits are very wide. ${ }^{19} 20$

\section{CONCLUSION}

Knowledge of the prognostic value of cranial ultrasound and MRI rests on a relatively small set of reports; often the meta-analysis could only be performed using two studies, and even then measures of heterogeneity were high. The values provided in this review are pragmatic rather than definitive. In particular, we did not feel that the data were sufficient to calculate separate estimates for different gestational ages, despite the large differences in prior probability of later problems over gestation. Nevertheless, for those who might wish to use age-specific priors (available from the Epipage study ${ }^{5}$ ) we provide likelihood ratios in table 1 .

However, these indicative results have clinical relevance. While a normal ultrasound scan confidently predicts a reduced risk of later motor problems, the prognostic values of many specific abnormalities detected by both ultrasound and MRI have wide confidence limits. In the recent paper by Horsch et al, infants with a normal cranial ultrasound scan had normal or mild white matter changes only on MRI. ${ }^{3}$ Together with the results of the study by Horsch et al, our review suggests that the risk of abnormal motor outcome is low and can be determined with some confidence for the majority of preterm infants, but is less certain in the $10-20 \%$ of infants with moderate abnormalities, irrespective of the imaging modality used. While some of this uncertainty is due to the small number of patients available for some analyses, the inherent imprecision of imaging and later childhood influences that affect neurodevelopment must also be contributing factors. Wise clinicians have always known that individual tests rarely provide certainty, and they continue to use neuroimaging with circumspection.

\section{Competing interests None.}

Provenance and peer review Commissioned; internally peer reviewed.

Arch Dis Child Fetal Neonatal Ed 2010;95:F388-F390. doi:10.1136/adc.2009.168997

\section{REFERENCES}

1. Tucker J, McGuire W. Epidemiology of preterm birth. BMJ 2004;329:675-8.

2. Hamilton BE, Miniño AM, Martin JA, et al. Annual summary of vital statistics: 2005. Pediatrics 2007:119:345-60.

3. Horsch S, Skiöld B, Hallberg B, et al. Cranial ultrasound and MRI at term age in extremely preterm infants. Arch Dis Child Fetal Neonatal Ed 2010;95:F310-14

4. Zamora J, Abraira V, Muriel A, et al. Meta-DiSc: a software for meta-analysis of test accuracy data. BMC Med Res Methodol 2006;6:31.

5. Larroque B, Ancel PY, Marret S, et al.; EPIPAGE Study group. Neurodevelopmental disabilities and special care of 5-year-old children born before 33 weeks of gestation (the EPIPAGE study): a longitudinal cohort study. Lancet 2008;371:813-20.

6. Papile LA, Burstein J, Burstein R, et al. Incidence and evolution of subependymal and intraventricular hemorrhage: a study of infants with birth weights less than 1,500 gm. J Pediatr 1978;92:529-34.

7. Volpe J. Neurology of the Newborn. Fourth edition. Philadelphia, PA: WB Saunders 2001.

8. De Vries LS, Van Haastert IL, Rademaker KJ, et al. Ultrasound abnormalities preceding cerebral palsy in high-risk preterm infants. J Pediatr 2004;144:815-20.

9. Ancel PY, Livinec F, Larroque B, et al.; EPIPAGE Study Group. Cerebral palsy among very preterm children in relation to gestational age and neonatal ultrasound abnormalities: the EPIPAGE cohort study. Pediatrics 2006;117:828-35.

10. Stewart AL, Thorburn RJ, Hope PL, et al. Ultrasound appearance of the brain in very preterm infants and neurodevelopmental outcome at 18 months of age. Arch Dis Child 1983;58:598-604.

11. Adams-Chapman I, Hansen NI, Stoll BJ, et al.; NICHD Research Network. Neurodevelopmental outcome of extremely low birth weight infants with posthemorrhagic hydrocephalus requiring shunt insertion. Pediatrics 2008;121:e1167-77.

12. Kuban KC, Allred EN, O'Shea TM, et al: ELGAN study investigators. Cranial ultrasound lesions in the NICU predict cerebral palsy at age 2 years in children born at extremely low gestational age. J Child Neurol 2009;24:63-72.

13. O'Shea TM, Kuban KC, Allred EN, et al.; Extremely Low Gestational Age Newborns Study Investigators. Neonatal cranial ultrasound lesions and developmental delays at 2 years of age among extremely low gestational age children. Pediatrics 2008;122:e662-9.

14. Patra K, Wilson-Costello D, Taylor HG, et al. Grades I-II intraventricular hemorrhage in extremely low birth weight infants: effects on neurodevelopment. J Pediatr 2006;149:169-73.

15. Stewart AL, Reynolds EO, Hope PL, et al. Probability of neurodevelopmental disorders estimated from ultrasound appearance of brains of very preterm infants. Dev Med Child Neurol 1987;29:3-11.

16. International PHVD Drug Trial Group. International randomised controlled trial of acetazolamide and furosemide in post haemorrhagic ventricular dilatation in infancy. Lancet 1998:352:433-40.

17. Whitelaw A, Jary S, Kmita G, et al. Randomized trial of drainage, irrigation and fibrinolytic therapy for premature infants with posthemorrhagic ventricular dilatation: developmental outcome at 2 years. Pediatrics 2010;125:e852-8.

18. Ventriculomegaly Trial Group. Randomised trial of early tapping in neonatal posthaemorrhagic ventricular dilation. Arch Dis Child 1990;65:3-10.

19. De Vries LS, Groenendaal F, van Haastert IC, et al. Asymmetrical myelination of the posterior limb of the internal capsule in infants with periventricular haemorrhagic infarction: an early predictor of hemiplegia. Neuropediatrics 1999:30:314-19

20. Roelants-van Rijn AM, Groenendaal F, Beek FJ, et al. Parenchymal brain injury in the preterm infant: comparison of cranial ultrasound, MRI and neurodevelopmental outcome. Neuropediatrics 2001;32:80-9

21. Woodward LJ, Anderson PJ, Austin NC, et al. Neonatal MRI to predict neurodevelopmental outcomes in preterm infants. N Engl J Med 2006; 355:685-94.

22. Mirmiran M, Barnes PD, Keller K, et al. Neonatal brain magnetic resonance imaging before discharge is better than serial cranial ultrasound in predicting cerebral palsy in very low birth weight preterm infants. Pediatrics 2004;114:992-8.

23. Miller SP, Ferriero DM, Leonard C, et al. Early brain injury in premature newborns detected with magnetic resonance imaging is associated with adverse early neurodevelopmental outcome. J Pediatr 2005:147:609-16. 